TITLE:

\title{
Effect of capacitive coupling in a miniature inductively coupled plasma source
}

\section{$\operatorname{AUTHOR}(S):$}

Takao, Yoshinori; Eriguchi, Koji; Ono, Kouichi

\section{CITATION:}

Takao, Yoshinori ... [et al]. Effect of capacitive coupling in a miniature inductively coupled plasma source. Journal of Applied Physics 2012, 112(9): 093306.

ISSUE DATE:

2012-11

URL:

http://hdl.handle.net/2433/161047

RIGHT:

(C) 2012 American Institute of Physics 


\section{AIP Appitied Physics}

\section{Effect of capacitive coupling in a miniature inductively coupled plasma source}

Yoshinori Takao, Koji Eriguchi, and Kouichi Ono

Citation: J. Appl. Phys. 112, 093306 (2012); doi: 10.1063/1.4764333

View online: http://dx.doi.org/10.1063/1.4764333

View Table of Contents: http://jap.aip.org/resource/1/JAPIAU/v112/i9

Published by the American Institute of Physics.

\section{Related Articles}

An alternative scaling model for neutron production in Z-pinch devices

Phys. Plasmas 19, 112702 (2012)

Microwave plasma source operating with atmospheric pressure air-water mixtures J. Appl. Phys. 112, 093301 (2012)

Intense plasma emission induced by jet-to-jet coupling in atmospheric pressure plasma arrays Appl. Phys. Lett. 101, 173503 (2012)

Numerical study of the characteristics of the ion and fast atom beams in an end-Hall ion source J. Appl. Phys. 112, 083301 (2012)

Boron-rich plasma by high power impulse magnetron sputtering of lanthanum hexaboride J. Appl. Phys. 112, 086103 (2012)

\section{Additional information on J. Appl. Phys.}

Journal Homepage: http://jap.aip.org/

Journal Information: http://jap.aip.org/about/about_the_journal

Top downloads: http://jap.aip.org/features/most_downloaded

Information for Authors: http://jap.aip.org/authors

\section{ADVERTISEMENT}
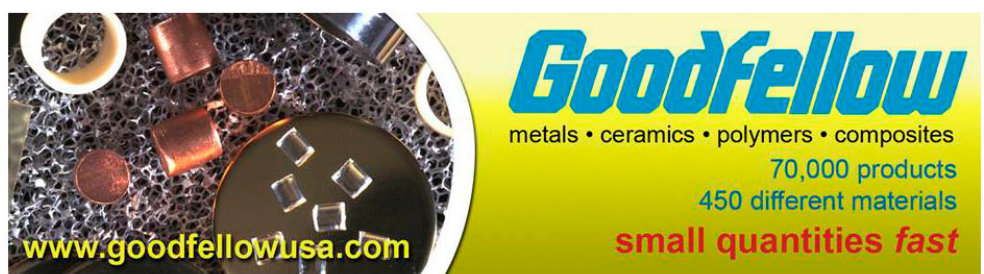


\title{
Effect of capacitive coupling in a miniature inductively coupled plasma source
}

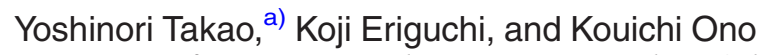 \\ Department of Aeronautics and Astronautics, Graduate School of Engineering, Kyoto University, \\ Yoshida-Honmachi, Sakyo-ku, Kyoto 606-8501, Japan
}

(Received 27 August 2012; accepted 9 October 2012; published online 7 November 2012)

\begin{abstract}
Two-dimensional axisymmetric particle-in-cell simulations with a Monte Carlo collision algorithm (PIC-MCC) have been conducted to investigate the effect of capacitive coupling in a miniature inductively coupled plasma source (mICP) by using two models: an inductive model and a hybrid model. The $\mathrm{mICP}$ is $3 \mathrm{~mm}$ in radius and $6 \mathrm{~mm}$ in height with a three-turn planar coil, where argon plasma is sustained. In the inductive model, the coil is assumed to be electrostatically shielded, and thus the discharge is purely inductive coupling. In the hybrid model, we assume that the different turns of the coil act like electrodes in capacitive discharge to include the effect of capacitive coupling. The voltage applied to these electrodes decreases linearly from the powered end of the coil towards the grounded end. The numerical analysis has been performed for $\mathrm{rf}$ frequencies in the range of $100-1000 \mathrm{MHz}$, and the power absorbed by the plasma in the range of $5-50 \mathrm{~mW}$ at a fixed pressure of 500 mTorr. The PIC-MCC results show that potential oscillations at the plasmadielectric interface are not negligible, and thus the major component of the absorbed power is caused by the axial motion of electrons in the hybrid model, although almost all of the power absorption is due to the azimuthal motion of electrons in the inductive model. The effect of capacitive coupling is more significant at lower rf frequencies and at higher absorbed powers under the calculation conditions examined. Moreover, much less coil currents are required in the hybrid model. @ 2012 American Institute of Physics. [http://dx.doi.org/10.1063/1.4764333]
\end{abstract}

\section{INTRODUCTION}

In order to broaden the application fields of microplasma sources, such as microthrusters, ${ }^{1-7}$ plasma displays, ${ }^{8,9}$ miniature mass spectrometers, ${ }^{10}$ and plasma biomedicine, ${ }^{11}$ it is crucial for a profound understanding of microplasma characteristics. For measurements of plasma parameters in a small space of less than a few millimeters the Langmuir probe method is very challenging, so optical diagnostics are widely used instead. ${ }^{9}$ However, the spatial distribution of plasma parameters is not readily available in experiments, and thus the information obtained from such experiments is limited. Since numerical simulations can also be useful to compensate for a lack of information, many fluid and particle simulations have already been performed..$^{1,5-7,12-16}$

In our previous study, two-dimensional axisymmetric particle-in-cell simulations with a Monte Carlo collision algorithm (PIC-MCC) were conducted to investigate the argon microplasma characteristics of a miniature inductively coupled plasma source $(\mathrm{mICP})$ with a $5 \mathrm{~mm}$ diameter planar coil, where the radius and length are $5 \mathrm{~mm}$ and $6 \mathrm{~mm}$, respectively. ${ }^{16}$ The numerical results were in reasonable agreement with the experimental data. In this model, however, capacitive coupling of the rf antenna coil to the plasma through a dielectric window was not taken into account. Hopwood et al. ${ }^{17,18}$ fabricated a mICP generator and experimentally investigated the plasma characteristic, which was to be expected in inductive discharges; their Langmuir probe measurements indicated

a)E-mail: takao.yoshinori.7a@kyoto-u.ac.jp. that the electron temperature and the plasma potential were independent of the rf power.

However, there should be a large potential difference between both coil ends since such mICPs were operated at a high frequency of $\sim 500 \mathrm{MHz} .{ }^{17-20}$ For example, if a mICP with a coil inductance $L=40 \mathrm{nH}$ is sustained by inducing the coil current $I_{\text {coil }}=1 \mathrm{~A}$ at the $\operatorname{rf}$ frequency $f=500 \mathrm{MHz}$, the potential drop reaches $126 \mathrm{~V}$ by calculating $2 \pi f L I_{\text {coil }}$. In conventional large ICPs, high electron densities $\left(10^{11}\right.$ $10^{12} \mathrm{~cm}^{-3}$ ) can be obtained, and thus the sheath thickness $(\sim 0.1 \mathrm{~mm})$ is much less than the thickness of the dielectric window (a few $\mathrm{cm}$ ). In this case, most of the potential can be dropped across the dielectric window, and thus capacitive coupling can be neglected. ${ }^{21}$ However, in mICPs the electron density is usually one order of magnitude lower than that in conventional ICPs, and the dielectric window is very thin (a few hundred $\mu \mathrm{m}){ }^{18-20}$ In this case, the sheath thickness becomes comparable to, or larger than, the dielectric window gap, and thus most of the large potential can be dropped across the sheath, implying that capacitive coupling would not be neglected if a Faraday shield is not inserted between the antenna and the plasma. Since the mICP developed by Hopwood et al. ${ }^{17-20}$ did not have a Faraday shield, their mICP might be sustained by capacitive discharges rather than inductive discharges. On the other hand, Doughty ${ }^{22}$ fabricated a mICP with a Faraday shield and showed that his mICP can operate with the shield. The difference between with and without a Faraday shield is to be investigated.

Although there are several papers on fluid simulations including capacitive coupling for conventional large ICPs, ${ }^{23-25}$ 
there are no papers on PIC-MCC simulations taking into account the effect of capacitive coupling for mICPs, to the best of our knowledge. In this work, we have incorporated the effect of capacitive coupling into our PIC-MCC model by using a simple method, which expresses the potential oscillation at the coil-dielectric interface. The purpose of this study is to investigate the effect of capacitive coupling in a mICP with a threeturn planar coil based on two models. The numerical model is described in Sec. II. The results and discussion are then presented in Sec. III, where we have indicated that most of the power deposition occurs in the axial direction when capacitive coupling is included, and the effect of capacitive coupling becomes more significant at lower frequencies and at higher absorbed powers. Finally, conclusions are drawn in Sec. IV.

\section{NUMERICAL MODEL}

Figure 1 shows a schematic of the mICP with the $5 \mathrm{~mm}$ diameter planar coil presently investigated, which has the same dimensions as that fabricated by Minayeva and Hopwood. ${ }^{19}$ The microplasma source is composed of a cylindrical metal tube the inner radius and the length of which are $3 \mathrm{~mm}$ and $6 \mathrm{~mm}$, respectively. The three-turn coil is located on the top of a $700 \mu \mathrm{m}$ thick dielectric window, and the bottom of the plasma source is bounded by a metal wall. The Ar plasma is generated by the rf current applied to the coil at high frequencies of 100-1000 MHz for a relatively high pressure of 500 mTorr, compared with conventional ICPs. The grid spacing is set at $0.1 \mathrm{~mm}$ at regular intervals, and the cylindrical coordinates $(r-z)$ are employed, with the origin being placed on the $z$-axis at the bottom of the plasma source.

The present PIC-MCC model consists of the electromagnetic equation for the rf-induced azimuthal electric field, Poisson's equation for the electrostatic field due to the space charge, the equation of motion, and collisions of charged

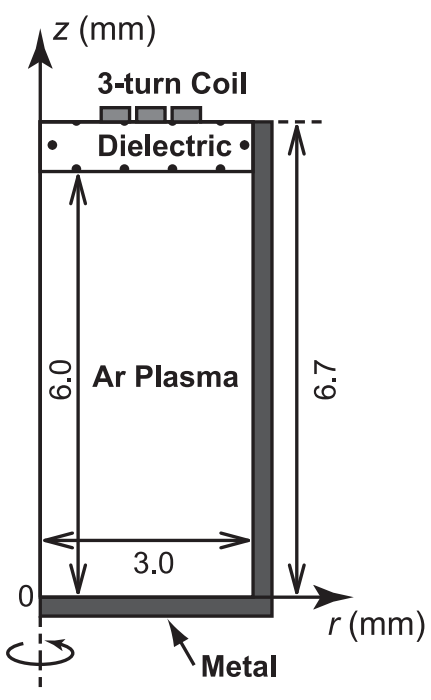

FIG. 1. Schematic of the mICP with the $5 \mathrm{~mm}$ diameter planar coil. The simulation area for charged particles is only the Ar plasma region $(6 \mathrm{~mm}$ in height and $3 \mathrm{~mm}$ in radius), while that for the potential is both the Ar plasma and dielectric regions $(6.7 \mathrm{~mm}$ in height and $3 \mathrm{~mm}$ in radius). The grid spacing is set at $0.1 \mathrm{~mm}$ at regular intervals, and cylindrical coordinates $(r-z)$ are employed, with the origin being placed on the $z$-axis at the bottom of the plasma source. particles. A number of simulated superparticles (singly ionized argon ions and electrons) are loaded into a twodimensional spatial computational mesh $(r, z)$, along with three velocity components $\left(v_{r}, v_{\theta}\right.$, and $\left.v_{z}\right)$. The equation of motion for charged particles is solved explicitly by a timecentered leap frog method for time integration and the Buneman-Boris method for the velocity advance with a coordinate rotation for the position advance. ${ }^{26}$ The collision of charged particles can be treated separately from the calculation of motion as long as the time step chosen is much smaller than the mean free time. ${ }^{27}$ The postcollision velocities of charged particles are determined by the conservation equations of momentum and energy. The reactions to be taken into account are elastic scattering, excitation, and ionization for electrons and elastic scattering and charge exchange for ions, ${ }^{28-31}$ which are described by the nullcollision method to reduce the calculation time. ${ }^{29}$ The data on the cross-sections for electron-neutral and ion-neutral collisions are the same as those used in Ref. 16. Neutral particles are assumed to be spatially uniform throughout the simulation with a Maxwellian velocity distribution at a gas temperature of $300 \mathrm{~K}$. The motion of excited-state atoms, Coulomb collisions between charged particles and the secondary electrons are not considered in this study. Our PICMCC model generally follows the methodology described in Ref. 16 and the references therein.

\section{A. Electromagnetic field}

The mICP is generated by the rf current applied to the antenna coil. All wave quantities, such as the electromagnetic field and current density, are assumed to vary harmonically in time as $e^{i \omega t}$, where $i$ is the square root of $-1, t$ is the time, and $\omega$ is the rf angular frequency. We also assume that the coil is composed of three concentric rings, so that the electric field only has the azimuthal component. Then, the induced electric field $E_{\theta}$ and the plasma current density $j_{\theta}$ are set to be $E_{\theta}=\tilde{E}_{\theta} e^{i \omega t}$ and $j_{\theta}=\tilde{j}_{\theta} e^{i \omega t}$, respectively, where $\tilde{E}_{\theta}$ and $\tilde{j}_{\theta}$ are the complex amplitudes. The complex amplitude of the electromagnetic fields is obtained from the following equation: ${ }^{32-35}$

$$
\left(\frac{\partial^{2}}{\partial r^{2}}+\frac{1}{r} \frac{\partial}{\partial r}+\varepsilon_{0} \mu_{0} \omega^{2}-\frac{1}{r^{2}}+\frac{\partial^{2}}{\partial z^{2}}\right) \tilde{E}_{\theta}=i \omega \mu_{0} \tilde{j}_{\theta},
$$

where $\varepsilon_{0}$ is the electric permittivity of a vacuum and $\mu_{0}$ is the magnetic permeability of a vacuum. The boundary conditions of $\tilde{E}_{\theta}$ are set to zero at the metal wall assuming perfectly conducting materials, and on the $z$-axis $(r=0)$ owing to the axisymmetry. On the plasma-dielectric window interfaces the electric field is analytically derived from BiotSavart's law, which is the sum of the fields over the threeturn coil current and the plasma current. ${ }^{33-36}$ The magnetic field $\mathbf{B}$ is then obtained from Faraday's law with the electric field determined by Eq. (1).

To solve Eq. (1) the relation between $\tilde{j}_{\theta}$ and $\tilde{E}_{\theta}$ should be specified. In the PIC-MCC model, one can derive the plasma current density directly by following electron trajectories. This calculation is fully kinetic and no assumptions 
are required about the mechanism of electron heating. The kinetic plasma current density is obtained from the following equation: ${ }^{37}$

$$
\tilde{j}_{\theta}=\frac{1}{V_{g}} \Sigma\left(-q W_{e} v_{\theta}\right) \exp (i \Delta \psi),
$$

where $q$ is the charge, $V_{g}$ is the cell volume centered at a grid point, $W_{e}$ is the weight of an electron superparticle (i.e., the number of physical electrons per computational particle for electrons), $v_{\theta}$ is the amplitude of the azimuthal component of the electron velocity at the fundamental frequency, $\Sigma$ is the summation of all the electron superparticles in the volume $V_{g}$, and $\Delta \psi$ is the phase difference between $I_{\text {coil }}$ and $j_{\theta}$. The azimuthal component of the ion current density can be ignored owing to the low mobility.

\section{B. Electrostatic field}

The potential $\phi$ and electrostatic field $\mathbf{E}$ due to the space charge are given by

$$
\begin{gathered}
\left(\frac{\partial^{2}}{\partial r^{2}}+\frac{1}{r} \frac{\partial}{\partial r}+\frac{\partial^{2}}{\partial z^{2}}\right) \phi=-\frac{\rho(r, z)}{\varepsilon_{0}}, \\
\mathbf{E}=-\nabla \phi
\end{gathered}
$$

where $\rho$ is the charge density. To solve Eq. (3) we choose to derive our finite differenced Poisson equation using a Gaussian pillbox on our rectangular mesh. ${ }^{38}$ The boundary conditions of $\phi$ are zero potential on the metal wall. On the plasma-dielectric interfaces, the surface charge $\sigma_{d}$ on the dielectric is also taken into account and obtained from the summation of the charged particles incident thereon. The electric fields on the plasma-dielectric boundaries are solved using half-size Gaussian pill boxes. ${ }^{38}$ It should be noted that the dielectric region is also included in the potential calculation, although the simulation area for charged particles is only the Ar plasma region.

\section{Capacitive coupling}

In order to investigate the effect of capacitive coupling of the rf antenna coil, we set two types of boundary condition on the top of the dielectric window $(z=6.7 \mathrm{~mm})$. Here, the dimensional effect of the coil is ignored and the current along the coil is assumed to be concentrated on the coildielectric interface. Figure 2 shows a schematic of the potential profile at the coil position $z=6.7 \mathrm{~mm}$ for both cases with and without the effect of capacitive coupling (w/ CC and w/o $\mathrm{CC}$ ). When capacitive coupling is not included, we assume that the coil is electrostatically shielded, and the potential is fixed at zero over the entire dielectric region with $z=6.7 \mathrm{~mm}$ as a boundary condition; thus, the discharge is purely inductive coupling. We refer to the analysis based on this as the inductive model (or expressed as "w/o CC" for short). On the other hand, when capacitive coupling is included, the innermost coil is assumed to be connected to an rf generator while the outermost coil is assumed to be connected to the ground. We also assume that the different turns of the coil

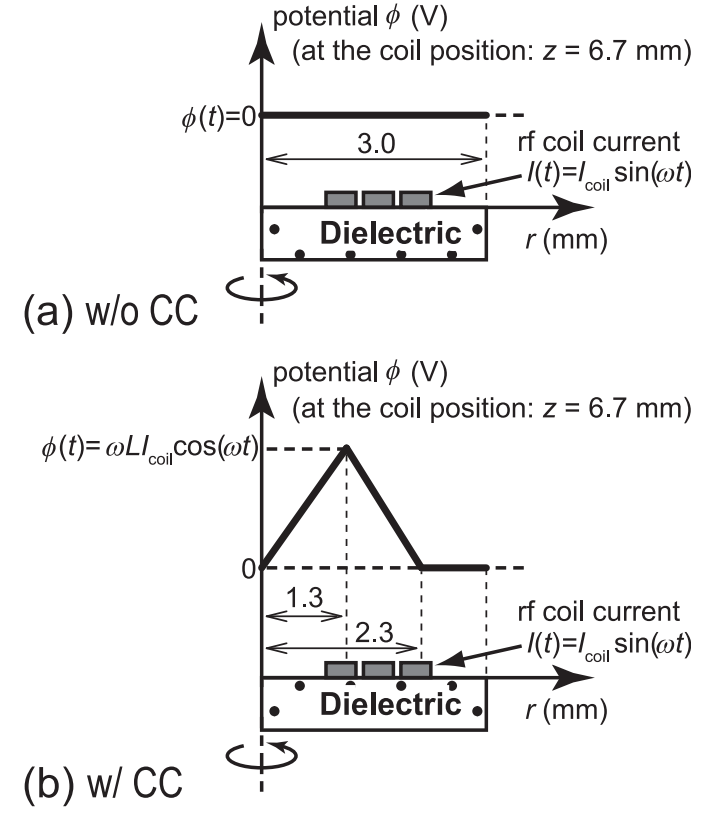

FIG. 2. Schematic of the potential profile on the top of the dielectric window $(z=6.7 \mathrm{~mm}$ ) for (a) the inductive model (without the effect of capacitive coupling: w/o CC) and (b) the hybrid model (with the effect of capacitive coupling: w/ CC). In the inductive model, the potential is fixed at zero as a boundary condition. In the hybrid model, the innermost coil is assumed to be connected to an rf generator while the outermost coil is assumed to be connected to the ground, and the voltage decreases linearly from the powered end of the coil $(r=1.3 \mathrm{~mm})$ towards the grounded end $(r=2.3 \mathrm{~mm})$ and the $z$-axis. Here, the dimensional effect of the coil is ignored, and the coil current flows at points $r=1.3,1.8$, and $2.3 \mathrm{~mm}$ on the coil-dielectric interface.

act like electrodes in capacitive discharge. The voltage applied to these electrodes decreases linearly from the powered end of the coil $(r=1.3 \mathrm{~mm})$ towards the grounded end $(r=2.3 \mathrm{~mm})$ and the $z$-axis. The potential between the outermost coil $(r=2.3 \mathrm{~mm})$ and the side metal wall $(r=3.0 \mathrm{~mm})$ is fixed at zero. Since the coil with inductance $L$ has the impedance of $i \omega L$, the potential at the innermost coil oscillates as $\phi(t)=\omega L I_{\text {coil }} \cos (\omega t)$ when the time-varying current of the rf coil is taken as $I(t)=I_{\text {coil }} \sin (\omega t)$. Here, the coil inductance $L$ is set at $36 \mathrm{nH}$ in our calculations. ${ }^{17}$ We refer to the analysis based on this as the hybrid model (or "w/ CC"). It should be noted that the above approximation would lead to an overestimation of capacitive coupling since $I_{\text {coil }}$ decreases along the coil owing to capacitive coupling between the coil and plasma. ${ }^{23,25}$ Implementation of this effect within our model is left for future work.

\section{Additional remarks}

Initially, spatially uniform ions and electrons with Maxwellian velocities are loaded in the simulation area, where the initial number of electron and ion superparticles is set at 550000 . When the number exceeds or falls below a predefined limit owing to their ionization or disappearance at the boundaries, the number of superparticles is adjusted to about the initial number in the same manner as described in Ref. 39. In order to speed up the simulation, ions motion and collisions are calculated only once per 25 electron time steps, owing to their difference in the speed of motion. Here, the numerical time step for electrons $\Delta t_{e}$ is taken to be 
$5.0 \times 10^{-12} \mathrm{~s}$ under every calculation condition presented in Sec. III. The time step is sufficient to resolve the electron plasma frequency and is much smaller than the mean free time in every case. The total power absorbed by the plasma $P_{a b s}$ is obtained by calculating the change in kinetic energy of electrons and ions before and after each charged particle is moved on integrating the equation of motion. ${ }^{40}$ In the simulation, the total power absorption is used as an input parameter; we rescale $I_{\text {coil }}$ to yield the specified total power absorption until the steady state solution is obtained. The macroscopic parameters, such as the electron density and electron temperature, are determined by averaging over $5 \mu \mathrm{s}$ after the steady state is reached.

\section{RESULTS AND DISCUSSION}

\section{A. Base case condition}

The PIC-MCC simulations for both the inductive and hybrid models were carried out at an Ar gas pressure $p=500$ mTorr, rf frequency $f=500 \mathrm{MHz}$, and absorbed power
$P_{a b s}=10 \mathrm{~mW}$ as the base case condition. It should be noted that the volume-averaged power density at $10 \mathrm{~mW}$ for the mICP shown in Fig. 1 is calculated to be $5.9 \times 10^{-2} \mathrm{~W} / \mathrm{cm}^{3}$, which is comparable to that for conventional ICPs. ${ }^{32}$ In such mICPs, the power transfer efficiency was determined to be less than a few percent below 1 Torr because most of the rf power was dissipated in the microfabricated circuit. ${ }^{19}$

Figure 3 shows the two-dimensional distribution of the absorbed power density $Q$, electron temperature $T_{e}$, electron density $n_{e}$, and potential $\phi$ in the mICP under the base case condition. The distribution of $Q$ in the inductive model is quite different from that in the hybrid model. In the inductive model, most of the rf power is deposited locally near the coil in a toroidal pattern, where the electrons are heated, and a negative power deposition can be seen in most of the bulk area because of the electron diffusion towards the walls, while a positive power deposition appears widely in the hybrid model. In both models, a positive power deposition on the walls is exhibited owing to the ion heating in the sheath area. Since electrons diffuse to the bulk region and

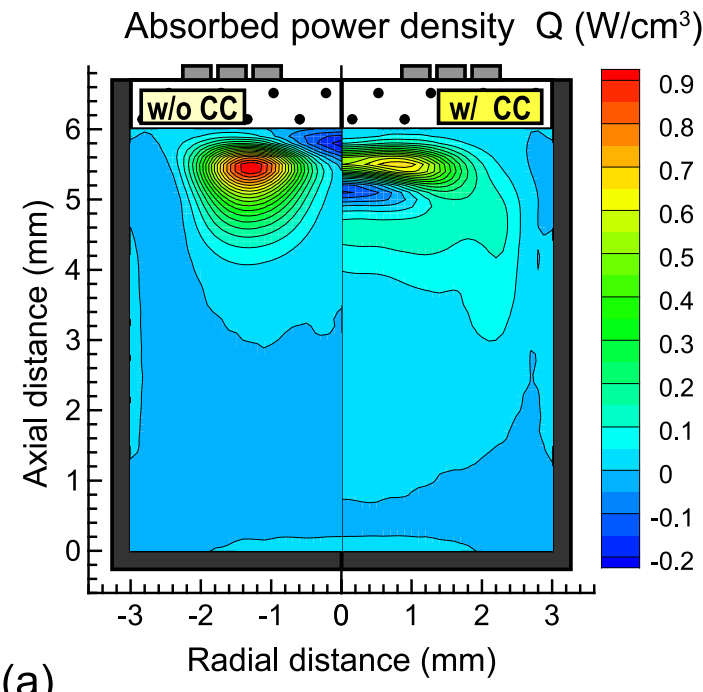

(a)

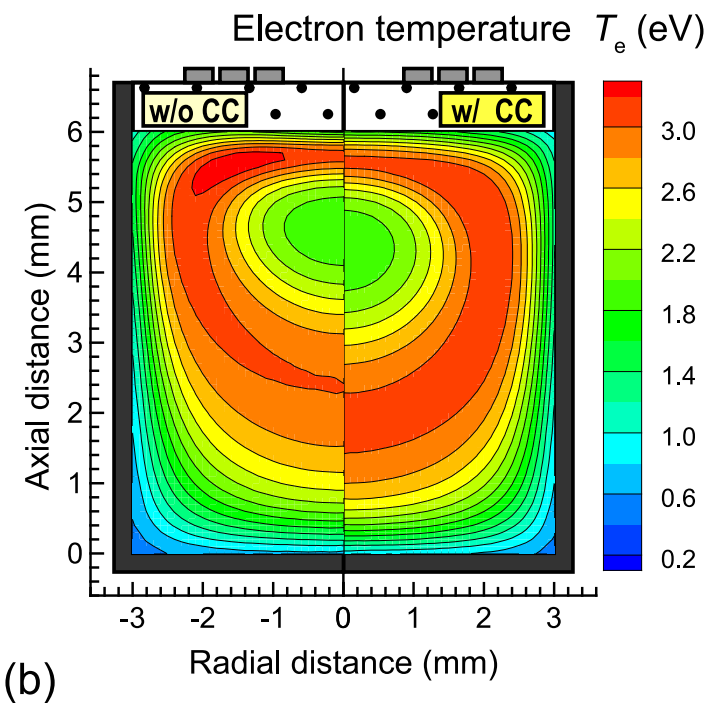

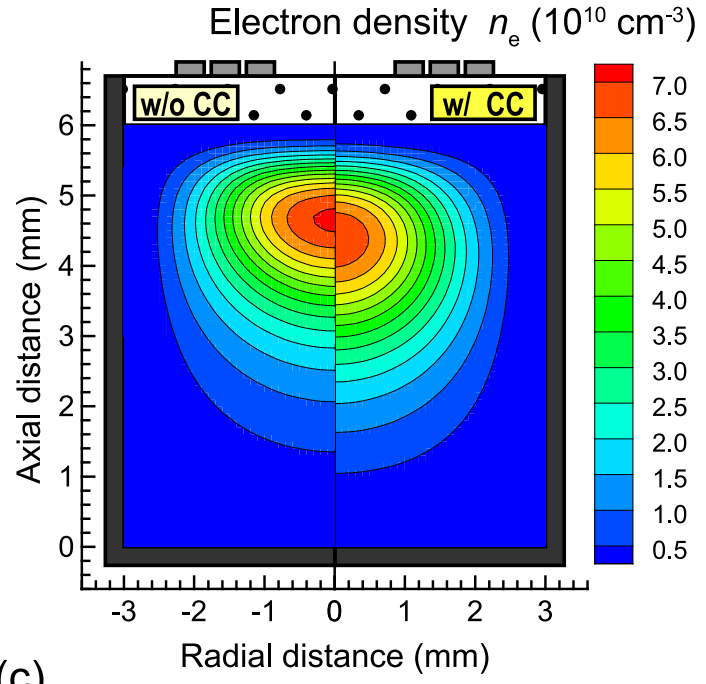

(c)

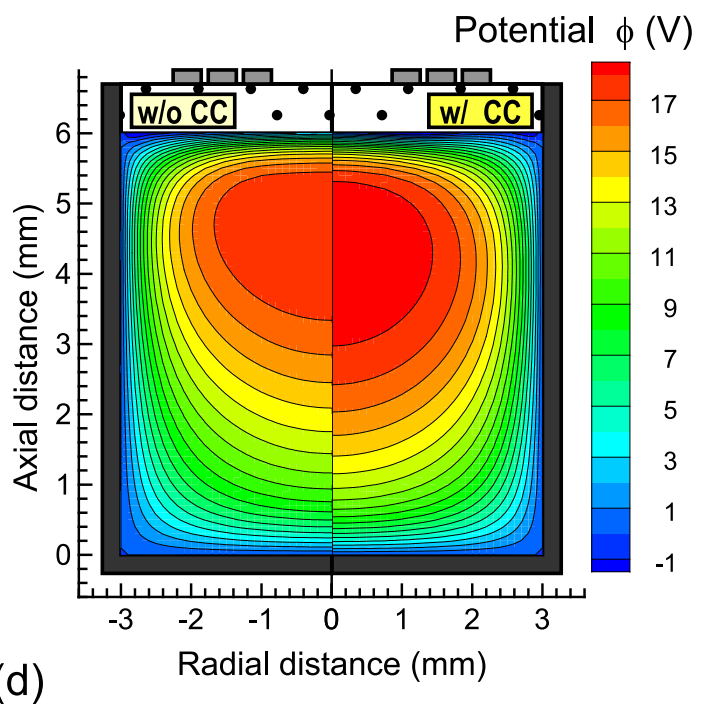

FIG. 3. Two-dimensional distributions of the time-averaged (a) absorbed power density $Q$, (b) electron temperature $T_{e}$, (c) electron density $n_{e}$, and (d) potential $\phi$ in the mICP based on the inductive model (left, w/o CC) and the hybrid model (right, w/ CC), calculated at the Ar gas pressure $p=500 \mathrm{mTorr}$, rf frequency $f=500 \mathrm{MHz}$, and absorbed power $P_{a b s}=10 \mathrm{~mW}$ as a base case condition. 
lose their energy through collisions, $T_{e}$ decreases around the center of the mICP, where the flat potential is obtained. The effect of the ambipolar diffusion increases $T_{e}$ near the plasmasheath interface and the deceleration of electrons decreases $T_{e}$ near the walls due to the potential barrier. The peak $T_{e}$ is obtained at a position close to where the peak $Q$ is seen in the inductive model, while there is no significant peak in the hybrid model and the high $T_{e}$ spreads to the bottom area compared to the inductive model. Although the gas pressure is relatively high, the peak $n_{e}$ is not localized where the peak $Q$ is obtained and exhibited on the $z$-axis close to the coil, owing to the diffusion effect and large surface-to-volume ratio of the mICP. In the hybrid model, the peak $n_{e}$ is a little away from the coil and its value $\left(6.9 \times 10^{10} \mathrm{~cm}^{-3}\right)$ is slightly lower than that in the inductive model $\left(7.1 \times 10^{10} \mathrm{~cm}^{-3}\right)$. While the distribution of $\phi$ is similar to that of $n_{e}$, the peak $\phi$ in the hybrid model $(18.8 \mathrm{~V})$ is slightly higher than that in the inductive model $(17.9 \mathrm{~V})$, which implies the effect of capacitive coupling. Compared with the difference of $Q$ distributions between both models, measurable parameters $\left(T_{e}, n_{\mathrm{e}}\right.$, and $\left.\phi\right)$ show little difference among their distributions. Therefore, it would be almost impossible in experiments to measure the difference in distributions obtained above.

Figure 4 shows the axial distributions of the potential $\phi$, electron density $n_{e}$, and ion density $n_{i}$ at the innermost coil $(r=1.3 \mathrm{~mm})$ in the mICP based on the hybrid model under the base case condition. The plots of $\phi$ and $n_{e}$ are shown at several times in the phase of the rf cycle: $\omega t / 2 \pi=0,1 / 8,2 / 8$, $4 / 8,5 / 8$, and $6 / 8$. Here, the ion is immobile during the rf cycle of $500 \mathrm{MHz}$, so that the profile of $n_{i}$ is plotted as a time-averaged value. Since the thickness of the dielectric window is comparable to the sheath thickness, as shown in Fig. 4(b), the amplitude of the potential oscillation at plasma-dielectric interface $(z=6.0 \mathrm{~mm})$ is about half of that at the three-turn coil $(z=6.7 \mathrm{~mm})$, and hence the effect of capacitive coupling is not negligible. As shown in the inset in Fig. 4(b), the value of $n_{e}$ becomes maximum at the phase $\omega t / 2 \pi=1 / 8$ and minimum at $5 / 8$, although the maximal and minimal values of $\phi$ at $z=6.7 \mathrm{~mm}$ are obtained at $\omega t / 2 \pi=0$ and $4 / 8$, respectively. Since electrons have inertia and the rf frequency is relatively high, electrons slightly lag the potential. Moreover, $\phi$ at $z=6.0 \mathrm{~mm}$ is asymmetric with respect to zero and its time-averaged value becomes slightly negative (see Fig. 10(b)). Since the net direct current should be zero on the dielectric wall, the dielectric window plays the role of a blocking capacitance for capacitive discharges.

Figure 5 shows the normalized electron energy probability function (EEPF) in the entire plasma area under the base case condition. Here, the normalized $\operatorname{EEPF} f_{p}(\varepsilon)$ is defined as $f_{p}(\varepsilon)=f_{d}(\varepsilon) / \sqrt{\varepsilon}$, where $\varepsilon$ is the electron energy in electron volt and $f_{d}(\varepsilon)$ is a normalized electron energy distribution function, i.e., $\int f_{d}(\varepsilon) d \varepsilon=1$. As shown in the figure, both the EEPFs are non-Maxwellian. At higher electron energies, depletions in EEPFs are clearly seen owing to the inelastic collisions, where the depletions occur above the excitation threshold energy $\left(E_{e x}=11.6 \mathrm{eV}\right)$. At lower electron energies, the EEPF of the inductive model is the same as that of the hybrid model, while the inductive model results in a large amount of high energy electrons compared with the
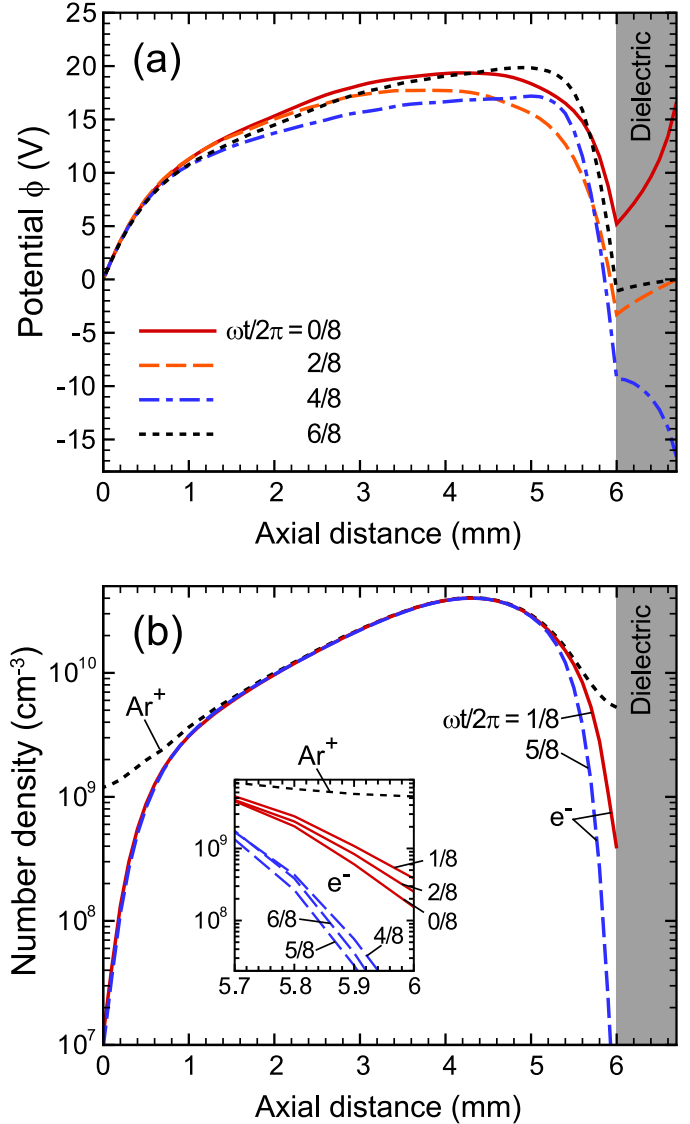

FIG. 4. Axial distributions of the (a) potential $\phi$, (b) electron density $n_{e}$ and ion density $n_{i}$ at the innermost coil $(r=1.3 \mathrm{~mm})$ in the mICP based on the hybrid model (w/ CC) under the same conditions as those in Fig. 3. Notice that the three-turn coil is located at the axial distance $z=6.7 \mathrm{~mm}$ as shown in Fig. 1. The plots of $\phi$ and $n_{e}$ are shown at several times in the phase of the rf cycle: $\omega t / 2 \pi=0,1 / 8,2 / 8,4 / 8,5 / 8$, and $6 / 8$, while $n_{i}$ is plotted as a time-averaged value. The inset shows the detailed profiles of $n_{e}$ and $n_{i}$ in the sheath region adjacent to the coil.

hybrid model. A high electron energy leads to a high ionization rate (as will be shown in Table I in the following Subsection III B), and then a high electron density in the inductive model as shown in Fig. 3(c).

\section{B. Frequency dependence}

Figure 6 shows the time-averaged distribution of $n_{e}$ and its peak values in the $\mathrm{mICP}$ as a function of rf frequency in the range of 100-1000 MHz. In the inductive model, the distribution of $n_{e}$ is almost independent of the frequency, where the peak $n_{e}$ occurs at around $z=4.7 \mathrm{~mm}$ on the $z$-axis under every frequency condition, while the peak $n_{e}$ slightly decreases with increasing frequency. In the hybrid model, the distribution of $n_{e}$ is dependent on the frequency and the peak $n_{e}$ significantly increases with an increase in frequency. The position of the peak $n_{e}$ moves away from the coil with decreasing frequency, implying a thicker sheath adjacent to the coil and less inductive coupling.

Figure 7 shows the axial distribution of the timeaveraged $\phi$ and the surface charge density on the dielectric window at the innermost coil $(r=1.3 \mathrm{~mm})$ for $f=100,250$, 500 , and $1000 \mathrm{MHz}$. The potential distribution is almost independent of the frequency in the inductive model, while 


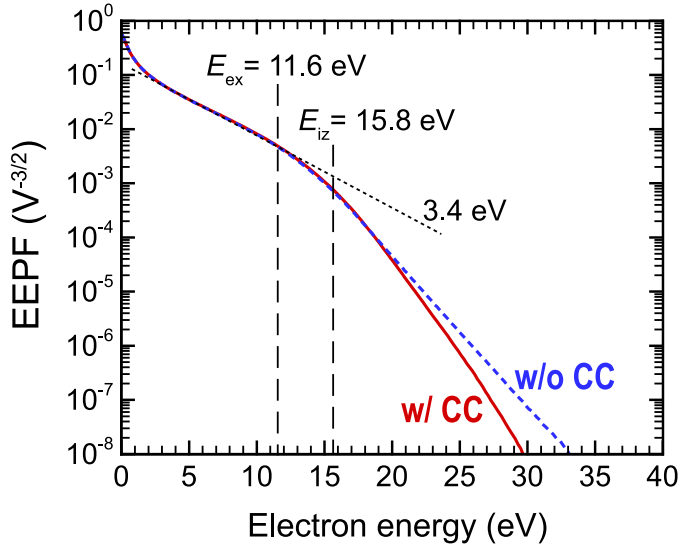

FIG. 5. Normalized EEPFs in the entire plasma area based on the inductive model (w/o CC) and the hybrid model (w/ CC) under the same conditions as those in Fig. 3. Here, $E_{e x}$ and $E_{i z}$ are the excitation and ionization threshold energies, respectively.

significant frequency dependence is observed in the hybrid model, where $\phi$ in the bulk area increases and $\phi$ at the plasmadielectric interface decreases and exhibits significantly negative values with decreasing frequency. At lower frequencies, more electrons reach the dielectric window during the positive cycle of the potential oscillation, so that the dielectric window is more negatively charged, which results in the negative potential on the dielectric. This large potential difference between in the bulk and on the dielectric leads to the large ion heating. Since a larger fraction of rf power deposits into ions through the acceleration in the sheath, which reduces the power deposition into electrons, $n_{e}$ decreases with decreasing frequency. In addition, the large negative potential increases the sheath thickness adjacent to the coil, which results in the distribution of $n_{e}$ as shown in Fig. 6(a).

Table I shows the coil current $I_{\text {coil }}$ and power balance results based on both models for $f=100,250,500$, and

TABLE I. Coil current $I_{\text {coil }}$ and power balance results based on the inductive model (w/o CC) and the hybrid model (w/ CC), calculated at $p=500$ mTorr and $P_{a b s}=10 \mathrm{~mW}$ for $f=100,250,500$, and $1000 \mathrm{MHz}$.

\begin{tabular}{|c|c|c|c|c|c|c|c|c|}
\hline \multirow[b]{2}{*}{$f(\mathrm{MHz})$} & \multicolumn{4}{|c|}{ Inductive model (w/o CC) } & \multicolumn{4}{|c|}{ Hybrid model (w/ CC) } \\
\hline & 100 & 250 & 500 & 1000 & 100 & 250 & 500 & 1000 \\
\hline$I_{\text {coil }}(\mathrm{A})$ & 21.3 & 10.0 & 6.63 & 5.28 & 6.09 & 0.69 & 0.15 & 0.051 \\
\hline$P_{a b s, e}(\mathrm{~mW})$ & 8.57 & 8.54 & 8.43 & 8.29 & 6.74 & 8.17 & 8.44 & 8.48 \\
\hline$P_{a b s, e, r}(\mathrm{~mW})$ & -1.65 & -1.37 & -1.08 & -1.00 & 0.39 & 0.72 & 2.73 & 3.08 \\
\hline$P_{a b s, e, z}(\mathrm{~mW})$ & 1.11 & 0.87 & 0.23 & -0.42 & 5.92 & 6.71 & 4.77 & 5.25 \\
\hline$P_{a b s, e, \theta}(\mathrm{mW})$ & 9.11 & 9.04 & 9.29 & 9.71 & 0.43 & 0.73 & 0.95 & 0.16 \\
\hline$P_{a b s, i}(\mathrm{~mW})$ & 1.43 & 1.46 & 1.57 & 1.71 & 3.26 & 1.84 & 1.56 & 1.52 \\
\hline$P_{a b s, i, r}(\mathrm{~mW})$ & 0.80 & 0.81 & 0.85 & 0.90 & 1.03 & 0.97 & 0.91 & 0.88 \\
\hline$P_{a b s, i, z}(\mathrm{~mW})$ & 0.63 & 0.66 & 0.72 & 0.82 & 2.23 & 0.87 & 0.66 & 0.64 \\
\hline$P_{a b s, i, \theta}(\mathrm{mW})$ & 0.00 & 0.00 & 0.00 & 0.00 & 0.00 & 0.00 & 0.00 & 0.00 \\
\hline$P_{l, e, \text { wall }}(\mathrm{mW})$ & 0.21 & 0.24 & 0.30 & 0.38 & 0.36 & 0.25 & 0.23 & 0.24 \\
\hline$P_{l, i, \text { wall }}(\mathrm{mW})$ & 0.19 & 0.21 & 0.26 & 0.31 & 0.54 & 0.28 & 0.23 & 0.23 \\
\hline$P_{l, e, \text { elas }}(\mathrm{mW})$ & 0.06 & 0.06 & 0.05 & 0.05 & 0.04 & 0.06 & 0.06 & 0.05 \\
\hline$P_{l, e, e x c}(\mathrm{~mW})$ & 7.00 & 6.88 & 6.58 & 6.20 & 4.90 & 6.45 & 6.80 & 6.78 \\
\hline$P_{l, e, i o n}(\mathrm{~mW})$ & 1.30 & 1.36 & 1.50 & 1.67 & 1.44 & 1.41 & 1.36 & 1.42 \\
\hline$P_{l, i, \text { elas }}(\mathrm{mW})$ & 0.34 & 0.35 & 0.36 & 0.39 & 0.77 & 0.43 & 0.37 & 0.36 \\
\hline$P_{l, i, c e x}(\mathrm{~mW})$ & 0.90 & 0.91 & 0.95 & 1.01 & 1.95 & 1.13 & 0.97 & 0.93 \\
\hline
\end{tabular}
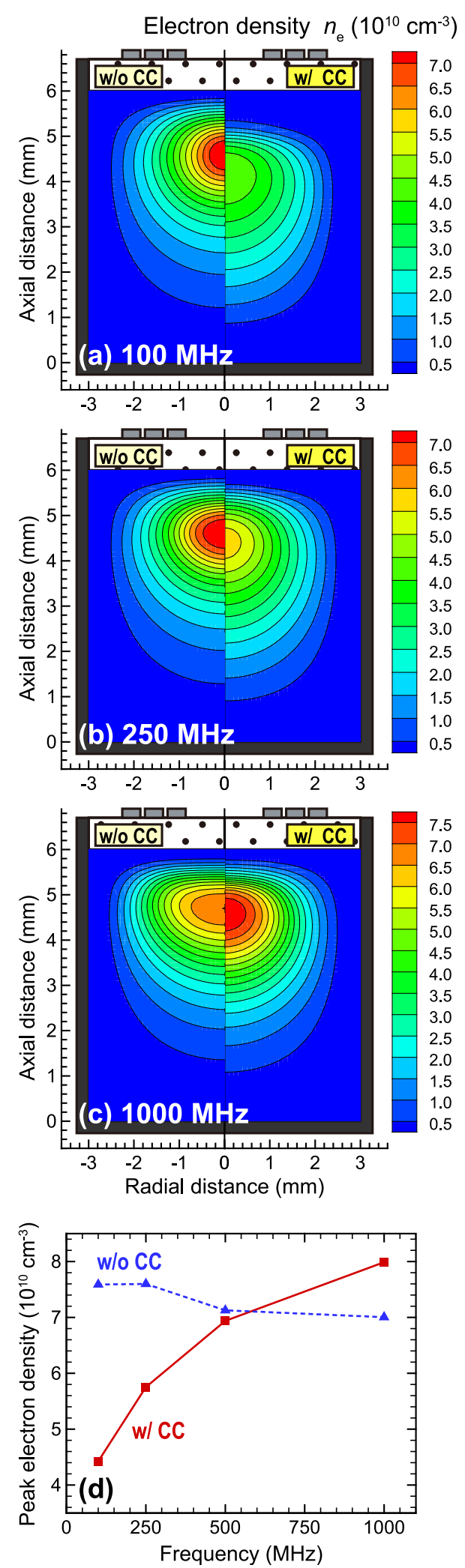

FIG. 6. Two-dimensional distributions of the time-averaged electron density $n_{e}$ in the mICP based on the inductive model (left, w/o CC) and the hybrid model (right, w/ CC) for $f=$ (a) 100 , (b) 250 , and (c) $1000 \mathrm{MHz}$; (d) the peak $n_{e}$ as a function of rf frequency, calculated at $p=500 \mathrm{mTorr}$ and $P_{a b s}=10 \mathrm{~mW}$.

$1000 \mathrm{MHz}$. The total power absorbed by the plasma $P_{a b s}$ consists of the electron heating $P_{a b s, e}$ and ion heating $P_{a b s, i}$, while the total power lost by the plasma $P_{\text {loss }}$ is equal to the sum of the power lost to electron and ion wall losses $\left(P_{l, e, \text { wall }}\right.$ 
and $P_{l, i, \text { wall }}$, respectively), and collisions with neutral particles. Here the collision losses are elastic scattering, excitation, and ionization for electrons $\left(P_{l, e, e l a s}, P_{l, e, e x c}\right.$, and $P_{l, e, i o n}$, respectively), and elastic scattering and charge exchange for ions ( $P_{l, i, e l a s}$ and $P_{l, i, c e x}$, respectively). The power absorbed by the plasma $P_{a b s}$ and the power lost by the plasma $P_{\text {loss }}$ agree to within $0.12 \%$. We also see similar good agreement between $P_{a b s, e}\left(=P_{a b s, e, r}+P_{a b s, e, z}+P_{a b s, e, \theta}\right)$ and $P_{l o s s, e} \quad\left(=P_{l, e, w a l l}\right.$ $\left.+P_{l, e, e l a s}+P_{l, e, e x c}+P_{l, e, i o n}\right)$ and between $P_{a b s, i}\left(=P_{a b s, i, r}\right.$ $\left.+P_{a b s, i, z}+P_{a b s, i, \theta}\right)$ and $P_{l o s s, i}\left(=P_{l, i, \text { wall }}+P_{l, i, e l a s}+P_{l, i, c e x}\right)$.
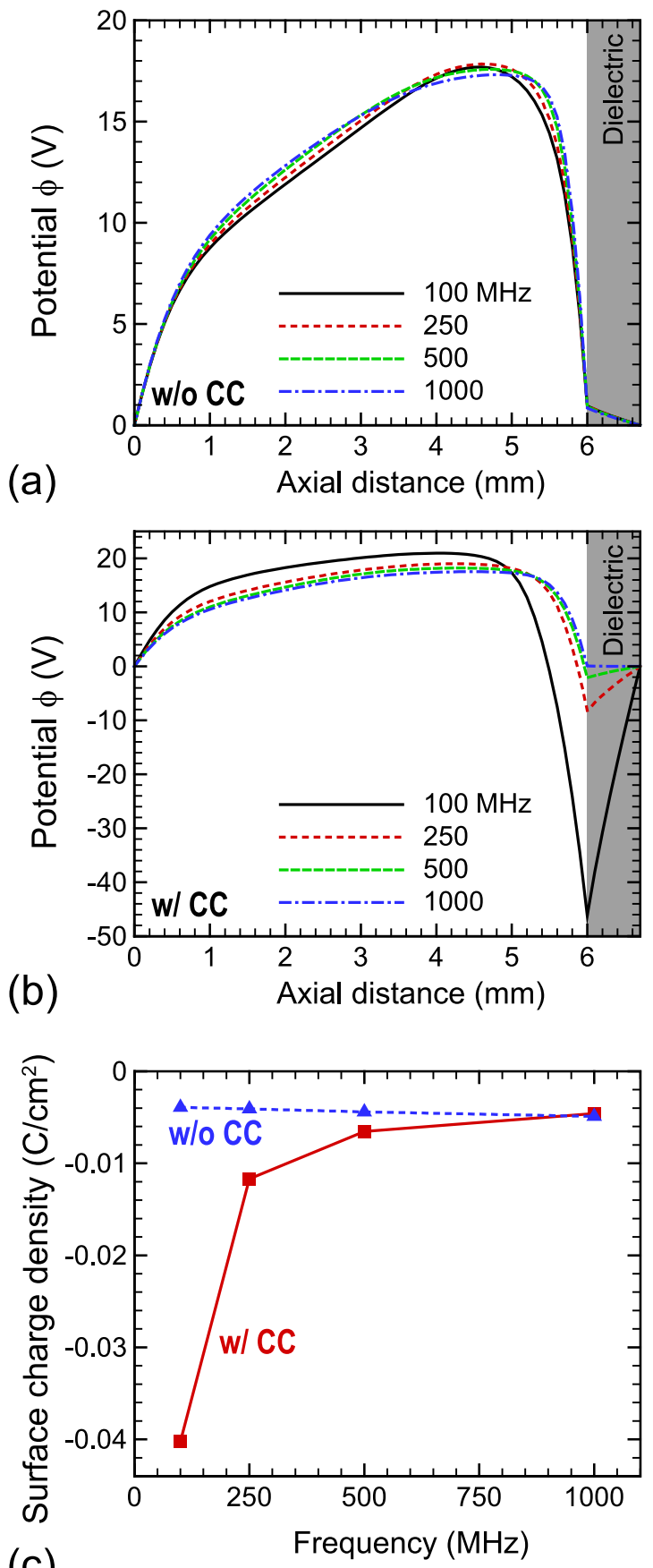

FIG. 7. Axial distributions of $\phi$ at the innermost coil $(r=1.3 \mathrm{~mm})$ in the mICP based on (a) the inductive model (w/o CC) and (b) the hybrid model (w/ CC); (c) the surface charge density on the dielectric window at $r=1.3 \mathrm{~mm}$ as a function of rf frequency under the same conditions as those in Fig. 6.
The absorbed power can be divided into each coordinate direction. In the inductive model, almost all of the power absorption is due to the azimuthal motion of electrons, while the major component of the power absorption is due to the axial motion of electrons in the hybrid model. This difference is clearly seen in the spatial distributions of the absorbed power density as show in Fig. 3(a). As indicated in Table I, capacitive coupling dominates over inductive coupling in the frequency range of $100-1000 \mathrm{MHz}$ in the hybrid model. Moreover, we confirm that a lot of rf power deposits into ions at lower frequencies, which is caused by the acceleration of the ions owing to the large potential drop in the sheath adjacent to the coil as discussed above. The large dependence of ion heating on the frequency results in a decrease in electron density with decreasing frequency. On the other hand, a substantial amount of power is lost through collisions, particularly due to the excitation of electrons owing to the high collision frequency at a high pressure of 500 mTorr.

Regarding the coil current required to deposit $10 \mathrm{~mW}$ into the plasma, Table I indicates that $I_{\text {coil }}$ in the inductive model is much higher than that in the hybrid model. Unfortunately, there are no experimental results of $I_{\text {coil }}$ for the mICP since the absolute measurement of $I_{\text {coil }}$ is difficult owing to the small size of the circuit and the inability to calibrate the current measurement at a high frequency of $500 \mathrm{MHz} .{ }^{19}$ Hence, we cannot directly compare our calculated $I_{\text {coil }}$ results with experimental data. In view of the fact that the coil of the mICP developed by Hopwood ${ }^{20}$ was fabricated from electroplated thin gold ( $7 \mu \mathrm{m}$ thickness and $400 \mu \mathrm{m}$ width), such a high current would be larger than the fusing current of a thin gold wire. ${ }^{41}$ This implies that his mICP would not be sustained by purely inductive discharges. It should be noted that at a frequency of $100 \mathrm{MHz}$, a high value $I_{\text {coil }}=6.09 \mathrm{~A}$ is required even in the hybrid model. The results of $I_{\text {coil }}$ indicate that it is desirable to use a thick and/ or wide antenna, to which a high current can be applied, when mICPs employ a Faraday shield or low frequency as demonstrated in Refs. 42-45.

Figure 8 shows the normalized EEPFs in the entire plasma area for $f=100,250,500$, and $1000 \mathrm{MHz}$. While a larger fraction of high energy electrons is obtained with increasing frequency in the inductive model, the number of high energy electrons tends to increase with decreasing frequency in the hybrid model. In the inductive model, most of the rf power is deposited into the electrons in the azimuthal direction, as indicated in Table I. Even though $I_{\text {coil }}$ decreases with increasing frequency, the azimuthal electric field increases with increasing frequency since the azimuthal electric field is proportional to the rf angular frequency. ${ }^{33,35}$ This higher electric field produces higher energy electrons. On the other hand, in the hybrid model, a higher $I_{\text {coil }}$ is also required with decreasing frequency as in the case of the inductive model. However, the increase rate is much larger than that of the rf frequency, so that a higher amplitude of the potential oscillation in the sheath is obtained at lower frequencies. Such a higher potential drop, i.e., a higher electric field, gives higher energy electrons at lower frequencies in the hybrid model. This actually leads to a higher ratio of 

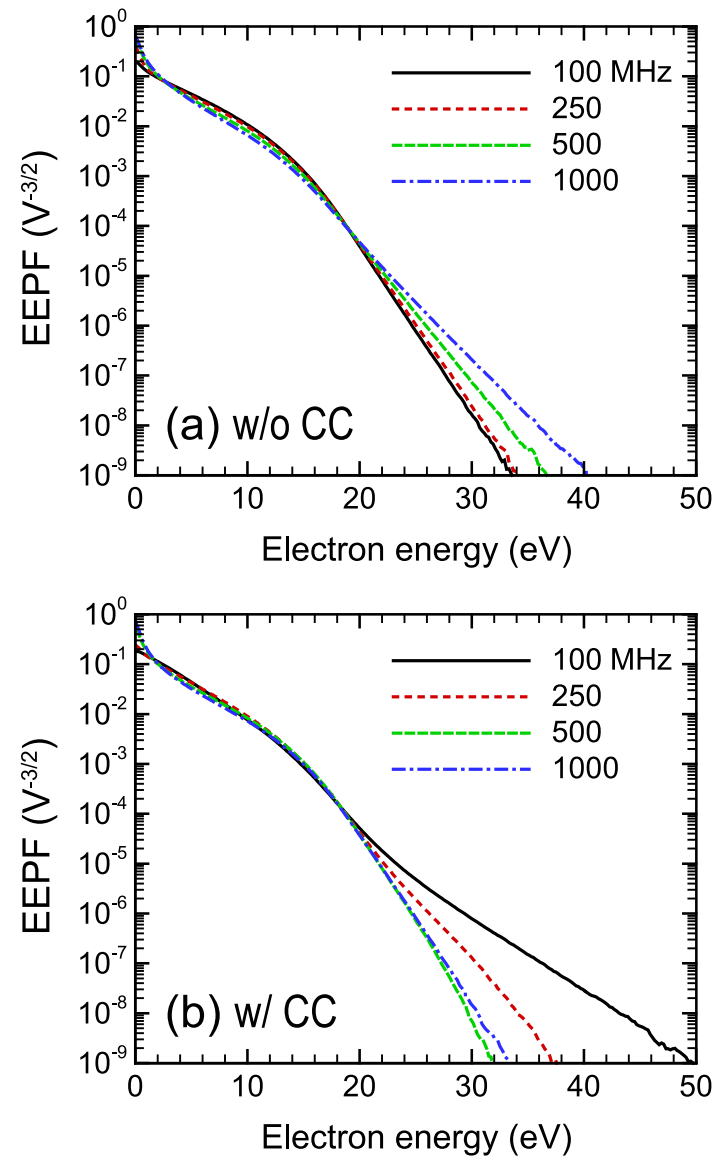

FIG. 8. Normalized EEPFs in the entire plasma area based on (a) the inductive model (w/o CC) and (b) the hybrid model (w/ CC) under the same conditions as those in Fig. 6.

ionization loss to the total collision loss at lower frequencies as shown in Table I. However, the wall loss is also higher with decreasing frequency, so that $n_{e}$ is lower than that in the inductive model at lower frequencies.

\section{Power dependence}

Figure 9 shows the time-averaged distribution of $n_{e}$ and its peak values in the mICP as a function of absorbed power in the range of 5-50 $\mathrm{mW}$. The peak $n_{e}$ increases linearly with increasing absorbed power in the inductive model, and its rate of increase is higher than that of the hybrid model. The electron density is more localized in the area near the coil in the inductive model while the distribution spreads over a wider area in the hybrid model. This would be due to the oscillation of the potential in the bulk plasma in the hybrid model as shown in Fig. 4(a).

Figure 10 shows the axial distribution of the timeaveraged $\phi$ and the surface charge density on the dielectric window at the innermost coil $(r=1.3 \mathrm{~mm})$ for $P_{a b s}=5,10$, 25 , and $50 \mathrm{~mW}$. Although the increase in $n_{e}$ results in a steep gradient of $\phi$ owing to the thinner sheath, the potential distributions remain almost the same in the inductive model as in the case of frequency dependence. In the hybrid model, the distribution of $\phi$ becomes flat in the bulk area with increasing absorbed power. The tendency is similar to that of the $n_{e}$ distribution as shown in Fig. 9. Increasing absorbed power
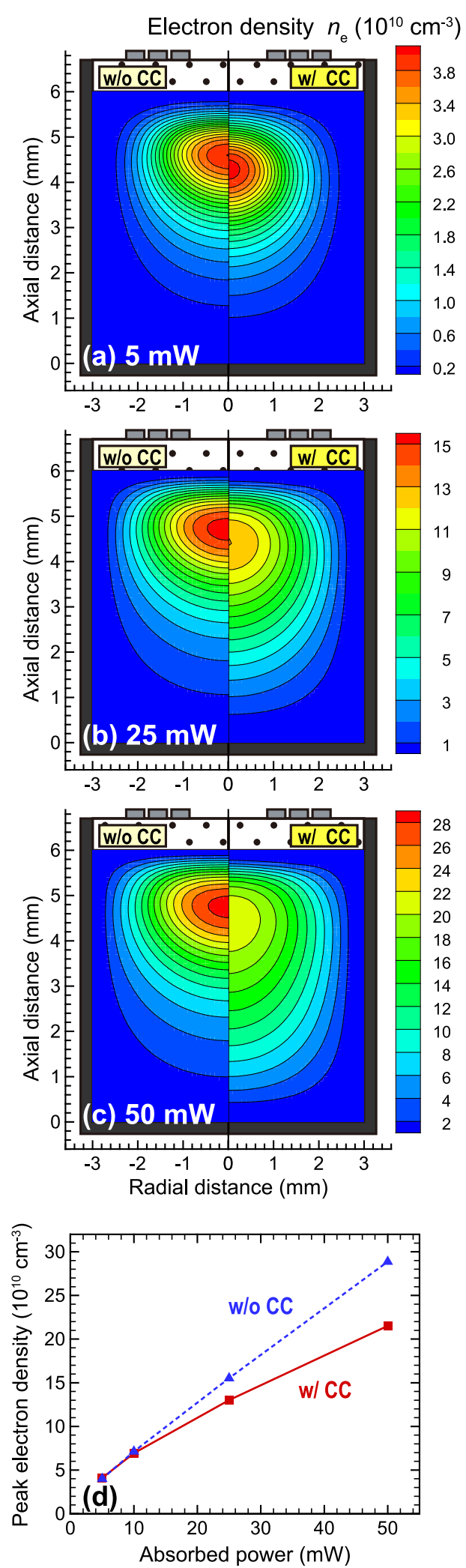

FIG. 9. Two-dimensional distributions of the time-averaged electron density $n_{e}$ in the mICP based on the inductive model (left, w/o CC) and the hybrid model (right, w/ CC) for $P_{a b s}=$ (a) 5 , (b) 25 , and (c) $50 \mathrm{~mW}$; (d) the peak $n_{e}$ as a function of absorbed power, calculated at $p=500 \mathrm{mTorr}$ and $f=500 \mathrm{MHz}$.

leads to an increase in large potential oscillation at the plasma-dielectric interface owing to the larger $I_{\text {coil }}$. This leads to the large negative charge on the dielectric window and large negative potential at the interface, and then a large 


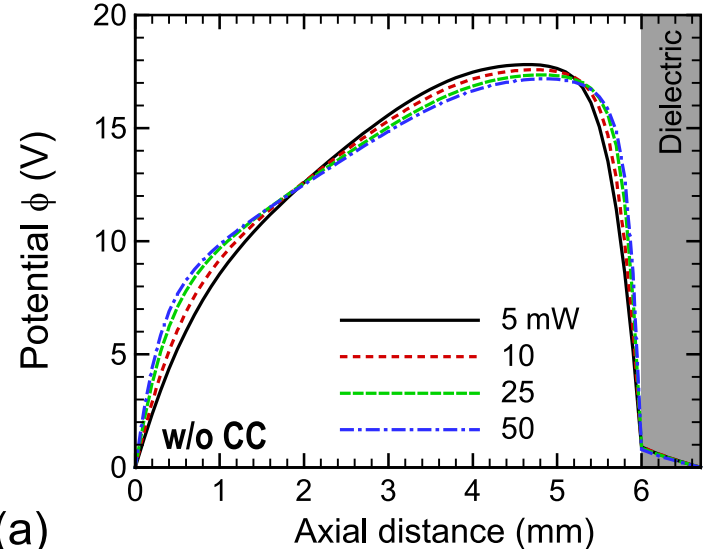

(a)

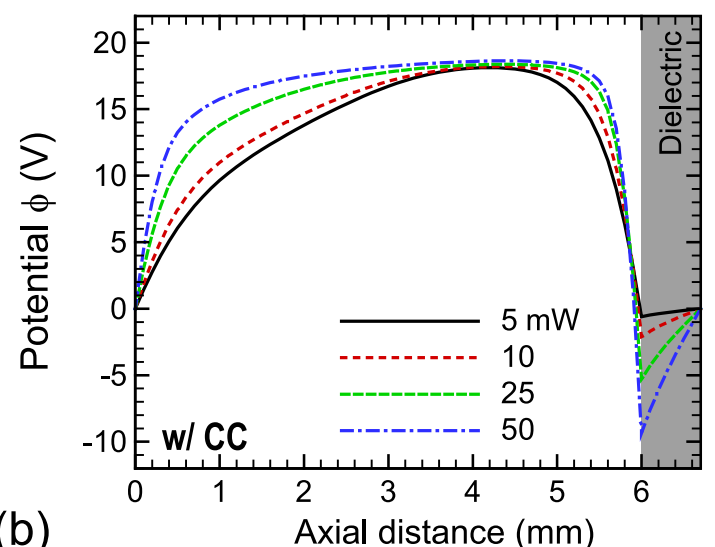

(b) Axial distance $(\mathrm{mm})$

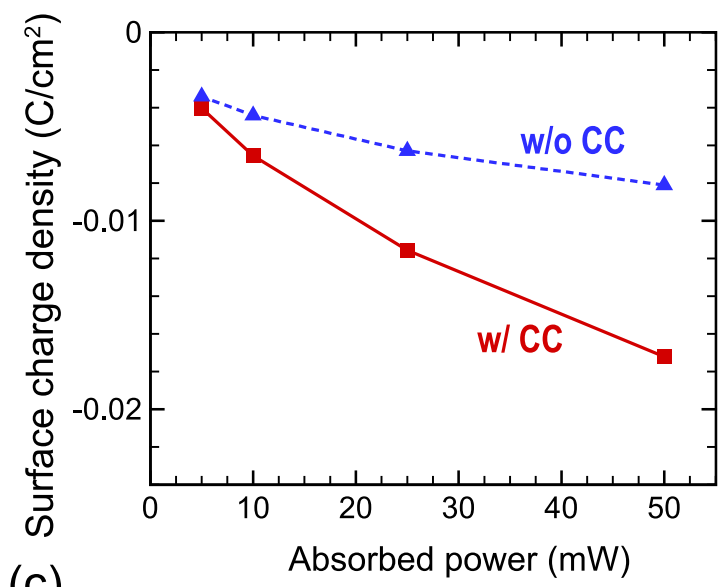

(c)

FIG. 10. Axial distributions of $\phi$ at the innermost coil $(r=1.3 \mathrm{~mm})$ in the mICP based on (a) the inductive model (w/o CC) and (b) the hybrid model (w/ CC); (c) the surface charge density on the dielectric window at $r=1.3 \mathrm{~mm}$ as a function of absorbed power under the same conditions as those in Fig. 9.

energy loss because of the undesired acceleration of the ions. This result can be seen in Table II, where $I_{\text {coil }}$ and power balance results are summarized. As shown in Table II, the percentage of the ion absorbed power increases, and that of the electrons decreases with increasing absorbed power in the hybrid model. Moreover, the higher rates of electron and ion losses to the walls occur at higher absorbed power. These effects suppress the increased rate of $n_{e}$ at higher absorbed powers in the hybrid model as shown in Fig. 9(d).

Figure 11 shows the normalized EEPFs in the entire plasma area for $P_{a b s}=5,10,25$, and $50 \mathrm{~mW}$, where the
TABLE II. Coil current $I_{c o i l}$ and power balance results based on the inductive model (w/o CC) and the hybrid model (w/ CC), calculated at $p=500$ mTorr and $f=500 \mathrm{MHz}$ for $P_{a b s}=5,10,25$, and $50 \mathrm{~mW}$. Note that each component of the power is expressed as a percentage of $P_{a b s}$.

\begin{tabular}{|c|c|c|c|c|c|c|c|c|}
\hline \multirow[b]{2}{*}{$P_{a b s}(\mathrm{~mW})$} & \multicolumn{4}{|c|}{ Inductive model (w/o CC) } & \multicolumn{4}{|c|}{ Hybrid model (w/ CC) } \\
\hline & 5 & 10 & 25 & 50 & 5 & 10 & 25 & 50 \\
\hline$I_{\text {coil }}(\mathrm{A})$ & 7.41 & 6.63 & 5.95 & 5.61 & 0.10 & 0.15 & 0.28 & 0.41 \\
\hline$P_{a b s, e} / P_{a b s}(\%)$ & 83.3 & 84.3 & 85.2 & 85.6 & 84.8 & 84.4 & 83.2 & 81.8 \\
\hline$P_{a b s, i} / P_{a b s}(\%)$ & 16.7 & 15.7 & 14.8 & 14.4 & 15.2 & 15.6 & 16.8 & 18.2 \\
\hline$P_{l, e, \text { wall }} / P_{a b s}(\%)$ & 3.36 & 2.95 & 2.62 & 2.45 & 2.08 & 2.26 & 2.52 & 2.75 \\
\hline$P_{l, i, w a l l} / P_{a b s}(\%)$ & 2.16 & 2.55 & 3.14 & 3.62 & 1.60 & 2.27 & 3.52 & 4.75 \\
\hline$P_{l, e, \text { elas }} / P_{a b s}(\%)$ & 0.48 & 0.53 & 0.58 & 0.61 & 0.54 & 0.55 & 0.56 & 0.56 \\
\hline$P_{l, e, e x c} / P_{a b s}(\%)$ & 63.7 & 65.8 & 67.7 & 68.5 & 68.8 & 68.0 & 65.9 & 64.1 \\
\hline$P_{l, e, i o n} / P_{a b s}(\%)$ & 15.8 & 15.0 & 14.3 & 14.0 & 13.5 & 13.6 & 14.2 & 14.5 \\
\hline$P_{l, i, \text { elas }} / P_{a b s}(\%)$ & 4.02 & 3.64 & 3.25 & 3.01 & 3.74 & 3.69 & 3.71 & 3.76 \\
\hline$P_{l, i, c e x} / P_{a b s}(\%)$ & 10.5 & 9.52 & 8.48 & 7.85 & 9.86 & 9.65 & 9.64 & 9.68 \\
\hline
\end{tabular}

opposite dependence on the absorbed power can be seen between the inductive model and the hybrid model. A larger fraction of high energy electrons is obtained with increasing absorbed power in the hybrid model, while high energy electrons tend to increase slightly with decreasing absorbed power in the inductive model. Hence, the ionization collision loss increases in the hybrid model and decreases in the inductive model with increasing absorbed power as indicated in
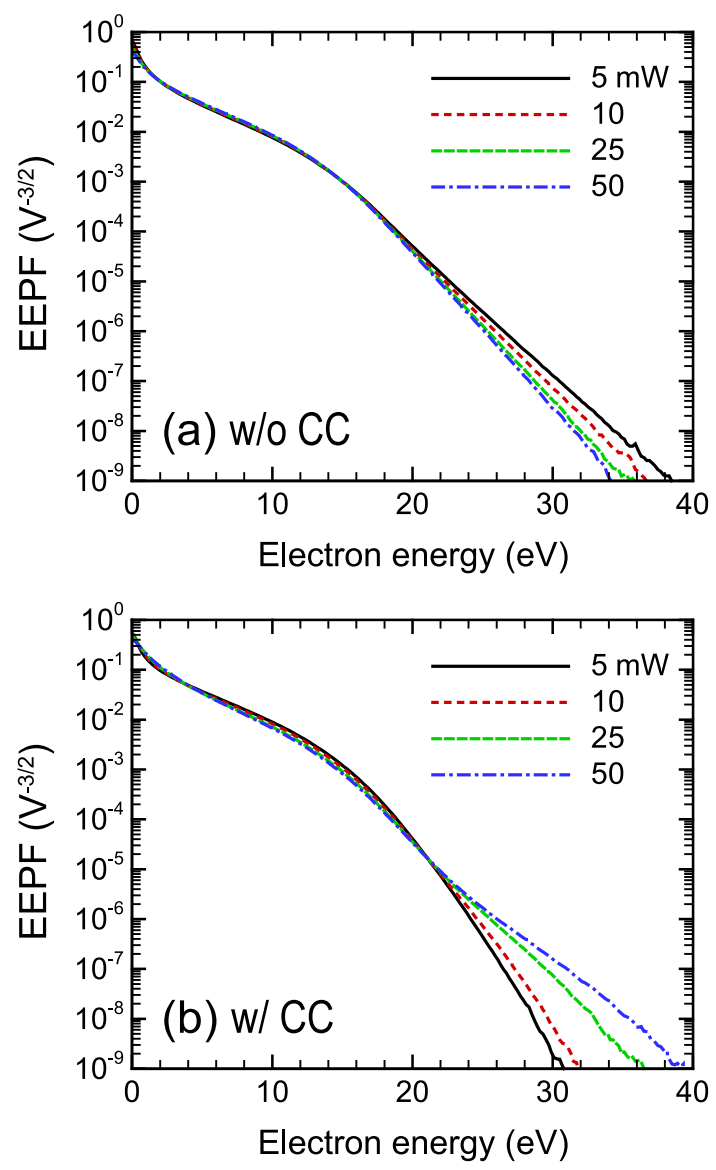

FIG. 11. Normalized EEPFs in the entire plasma area based on (a) the inductive model (w/o CC) and (b) the hybrid model (w/ CC) under the same conditions as those in Fig. 9. 
Table II. In the inductive model, $I_{\text {coil }}$ decreases with increasing absorbed power while $I_{\text {coil }}$ increases in the hybrid model. Since the electrons gain the energy from the azimuthal electric field in the inductive model and the azimuthal electric field is proportional to $I_{\text {coil }}$, high energy electrons decrease at higher absorbed power. On the other hand, in the hybrid model, a larger $I_{\text {coil }}$ is required with increasing absorbed power, and thus high energy electrons increase owing to the larger potential oscillation at the plasma-dielectric interface. It should be noted that a much larger $I_{\text {coil }}$ is required at the beginning of the calculation for higher power absorption in the inductive model. Once $n_{e}$ increases, the coupling efficiency between the rf electric field and the plasma increases, so that $I_{\text {coil }}$ decreases with increasing absorbed power at steady state.

\section{CONCLUSIONS}

Two-dimensional axisymmetric PIC-MCC simulations for a mICP in Ar have been carried out to investigate the effect of capacitive coupling by using an inductive model and a hybrid model. The mICP has a $3 \mathrm{~mm}$ inner radius and is $6 \mathrm{~mm}$ in height with a planar three-turn coil $(5 \mathrm{~mm}$ in diameter). Both inductive and capacitive coupling of the rf coil to the plasma through a dielectric window are included in the hybrid model, while the discharge is purely inductive coupling in the inductive model. The calculations have been performed for $\mathrm{rf}$ frequencies in the range of $100-1000 \mathrm{MHz}$, and the power absorbed by the plasma in the range of $5-50 \mathrm{~mW}$ at a fixed pressure of $500 \mathrm{mTorr}$.

The PIC-MCC results show that potential oscillation at the plasma-dielectric interface cannot be neglected in the hybrid model, since the thin dielectric window is comparable to the sheath thickness. Consequently, the major component of the power deposition is attributed to the axial motion of electrons and capacitive coupling dominates over inductive coupling in the hybrid model, whereas most of the power deposition is due to the azimuthal motion of electrons in the inductive model. The effect of capacitive coupling is more significant at lower $\mathrm{rf}$ frequencies and at higher absorbed powers, although there is slight difference under the base case condition $(500 \mathrm{MHz}, 10 \mathrm{~mW})$. The peak electron density significantly decreases with a decrease in frequency and its increasing rate decreases with increasing absorbed power in the hybrid model. This is due to the large negative charge on the dielectric window, which results in a large energy loss owing to undesired acceleration of ions in the sheath adjacent to the coil. In order to deposit a fixed power into the plasma, a much higher coil current is required in the case without capacitive coupling or at low frequency. This result indicates that it is desirable to use a thick and/or wide antenna, which has a large fusing current, when mICPs employ a Faraday shield or low frequency. The next steps include incorporating an external circuit, such as a matching network, into the present model.

\section{ACKNOWLEDGMENTS}

This work was financially supported in part by a Grantin-Aid for Scientific Research on Innovative Areas (Grant
No. 21110008) from the Ministry of Education, Culture, Sports, Science and Technology. Y.T. was supported by a Grant-in-Aid for Young Scientists (B) (Grant No. 23760769) from the Japan Society for the Promotion of Science.

${ }^{1}$ Y. Takao and K. Ono, Plasma Sources Sci. Technol. 15, 211 (2006).

${ }^{2}$ Y. Takao, K. Ono, K. Takahashi, and K. Eriguchi, Jpn. J. Appl. Phys. 45, 8235 (2006).

${ }^{3}$ Y. Takao, K. Eriguchi, and K. Ono, J. Appl. Phys. 101, 123307 (2007).

${ }^{4}$ Y. Takao, T. Takahashi, K. Eriguchi, and K. Ono, Pure Appl. Chem. 80, 2013 (2008).

${ }^{5}$ T. Takahashi, Y. Takao, K. Eriguchi, and K. Ono, Phys. Plasmas 16, 083505 (2009).

${ }^{6}$ T. Deconinck, S. Mahadevan, and L. L. Raja, J. Appl. Phys. 106, 063305 (2009).

${ }^{7}$ T. Takahashi, Y. Takao, Y. Ichida, K. Eriguchi, and K. Ono, Phys. Plasmas 18, 063505 (2011).

${ }^{8}$ K. H. Becker, K. H. Schoenbach, and J. G. Eden, J. Phys. D 39, R55 (2006).

${ }^{9}$ K. Tachibana, IEEJ Trans. Electr. Electron. Eng. 1, 145 (2006).

${ }^{10}$ R. Foest, M. Schmidt, and K. Becker, Int. J. Mass Spectrom. 248, 87 (2006).

${ }^{11}$ F. Iza, G. J. Kim, S. M. Lee, J. K. Lee, J. L. Walsh, Y. T. Zhang, and M. G. Kong, Plasma Process. Polym. 5, 322 (2008).

${ }^{12}$ J. Choi, F. Iza, J. K. Lee, and C. M. Ryu, IEEE Trans. Plasma Sci. 35, 1274 (2007).

${ }^{13}$ Y. Ikeda, K. Suzuki, H. Fukumoto, J. P. Verboncoeur, P. J. Christenson, C. K. Birdsall, M. Shibata, and M. Ishigaki, J. Appl. Phys. 88, 6216 (2000).

${ }^{14}$ S. K. Nam and D. J. Economou, J. Appl. Phys. 95, 2272 (2004).

${ }^{15}$ F. Iza, J. K. Lee, and M. G. Kong, Phys. Rev. Lett. 99, 075004 (2007).

${ }^{16}$ Y. Takao, N. Kusaba, K. Eriguchi, and K. Ono, J. Appl. Phys. 108, 093309 (2010).

${ }^{17}$ Y. Yin, J. Messier, and J. A. Hopwood, IEEE Trans. Plasma Sci. 27, 1516 (1999).

${ }^{18}$ J. Hopwood, O. Minayeva, and Y. Yin, J. Vac. Sci. Technol. B 18, 2446 (2000).

${ }^{19}$ O. B. Minayeva and J. Hopwood, J. Appl. Phys. 94, 2821 (2003).

${ }^{20}$ J. A. Hopwood, J. Microelectromech. Syst. 9, 309 (2000).

${ }^{21}$ M. A. Lieberman and A. J. Litchenberg, Principles of Plasma Discharges and Materials Processing, 2nd ed. (Wiley, Hoboken, NJ, 2005), p. 471.

${ }^{22}$ F. C. Doughty, U.S. patent 7,309,842 (2007).

${ }^{23}$ E. Kawamura, D. B. Graves, and M. A. Lieberman, Plasma Sources Sci. Technol. 20, 035009 (2011).

${ }^{24}$ T. Panagopoulos, D. Kim, V. Midha, and D. J. Economou, J. Appl. Phys. 91, 2687 (2002).

${ }^{25}$ E. F. Jaeger, L. A. Berry, J. S. Tolliver, and D. B. Batchelor, Phys. Plasmas 2, 2597 (1995).

${ }^{26}$ C. K. Birdsall and A. B. Langdon, Plasma Physics via Computer Simulation (IOP Publishing, Bristol, U.K., 1991), p. 338.

${ }^{27}$ K. Nanbu, IEEE Trans. Plasma Sci. 28, 971 (2000).

${ }^{28}$ A. Bogaerts, R. Gijbels, and W. Goedheer, Jpn. J. Appl. Phys. 38, 4404 (1999).

${ }^{29}$ V. Vahedi and M. Surendra, Comput. Phys. Commun. 87, 179 (1995).

${ }^{30}$ M. Surendra, D. B. Graves, and G. M. Jellum, Phys. Rev. A 41, 1112 (1990).

${ }^{31}$ W. H. Cramer, J. Chem. Phys. 30, 641 (1959).

${ }^{32}$ H. Takekida and K. Nanbu, IEEE Trans. Plasma Sci. 34, 973 (2006).

${ }^{33}$ B. W. Yu and S. L. Girshick, J. Appl. Phys. 69, 656 (1991).

${ }^{34}$ X. Chen and E. Pfender, Plasma Chem. Plasma Process. 11, 103 (1991).

${ }^{35} \mathrm{H}$. Fukumoto, I. Fujikake, Y. Takao, K. Eriguchi, and K. Ono, Plasma Sources Sci. Technol. 18, 045027 (2009).

${ }^{36} \mathrm{P}$. Silvester, Modern Electromagnetic Fields (Prentice-Hall, Englewood Cliffs, 1968), p. 153.

${ }^{37}$ S. Rauf and M. J. Kushner, J. Appl. Phys. 81, 5966 (1997).

${ }^{38}$ V. Vahedi and G. DiPeso, J. Comput. Phys. 131, 149 (1997).

${ }^{39}$ K. Nanbu and S. Kondo, Jpn. J. Appl. Phys. 36, 4808 (1997).

${ }^{40}$ M. Surendra and D. B. Graves, IEEE Trans. Plasma Sci. 19, 144 (1991).

${ }^{41}$ I. Bahl, Fundamentals of RF and Microwave Transistor Amplifiers (Wiley, Hoboken, NJ, 2009), p. 145.

${ }^{42}$ T. Ichiki, T. Koidesawa, and Y. Horiike, Plasma Sources Sci. Technol. 12, S16 (2003).

${ }^{43}$ T. Ichiki, R. Taura, and Y. Horiike, J. Appl. Phys. 95, 35 (2004).

${ }^{44}$ T. Ito, H. Nishiyama, K. Terashima, K. Sugimoto, H. Yoshikawa, H. Takahashi, and T. Sakurai, J. Phys. D 37, 445 (2004).

${ }^{45}$ S. Kumagai, H. Matsuyama, Y. Yokoyama, M. Hori, and M. Sasaki, Jpn. J. Appl. Phys. 50, 08JA02 (2011). 